\title{
ANALYSIS OF INDUCED OUANTITATIVE VARIATION I. HIERARCHICAL AND TRIPLE TEST CROSS ANALYSIS
}

\author{
D. S. VIRK*, G. S. POONI, N. R. INGRAM and J. L. JINKS \\ Department of Genetics, University of Birmingham, Birmingham B15 2TT, U.K.
}

Received 19.vi.81

\section{SUMMARY}

The triple test cross and a hierarchical inbreeding design are compared for their abilities to detect and analyse the quantitative variation induced in an $\mathrm{F}_{2}$ population by $\gamma$ radiation. Both designs detected an increase in the additive genetical variation for four contrasting characters in the cross of varieties 1 and 5 of Nicotiana rustica following irradiation of the $\mathrm{F}_{2}$ seed, but it was smaller in the triple test cross than in the hierarchical design. Because, however, the latter is based on inbreeding it provided no information on non-additive genetical variation in contrast to the triple test cross that detected a significant increase in the dominance variation.

The differences between the estimates of the additive genetic variance from the two designs can be largely accounted for by the differing contributions of repulsion linkages and the confounded effects of the unequal gene frequencies and multiple allelism created by the induced mutations in the rank 1 variances of the hierarchical design. The dominance contribution of loci at which multiple allelism has been induced may also be a contributing factor. Irrespective of the cause, however, the evidence for increased additive genetic variation following irradiation is unambiguous and it should lead to greater variation among the pure breeding lines which are in the process of being extracted from the irradiated $\mathrm{F}_{2}$.

\section{INTRODUCTION}

THE hierarchical biometrical genetical analysis of Virk, et al. (1978) and the $F_{2}$ triple test cross analysis of Kearsey and Jinks (1968) are in theory the most reliable methods for detecting induced quantitative variation in the generations immediately following a mutagenic treatment. In this paper we compare their abilities to detect, measure and analyse the variation in an $\mathrm{F}_{2}$ of the cross between pure breeding varieties 1 and 5 of Nicotiana rustica following irradiation of the $F_{2}$ seed.

\section{THEORY}

Virk et al. (1978) gave general expectations for the generations derived by selfing a pure breeding line following a mutagenic treatment. Where, as in the present experiment, an $F_{2}$ is treated it becomes equivalent to the $M_{1}$ generation of their model. The expectations, assuming an additive, dominance model, for the variances of the $F_{3}$ and $F_{4}$ generations obtained from an unirradiated control $F_{2}$ and from the $\mathrm{RF}_{3}$ and $\mathrm{RF}_{4}$ generations obtained from an irradiated $\mathrm{F}_{2}$ are given in table 1 . In Virk et al., analysis the highest ranking variances, in this case $V_{1 \mathrm{RF}_{3}}$ and $V_{1 \mathrm{RF}_{4}}$, are either omitted

* Present address: Department of Plant Breeding, Punjab Agricultural University, Ludhiana, India. 
TABLE 1

Generalised genetical expectations for the variances obtainable for the $F_{3}, R F_{3}, F_{4}$ and $\mathrm{RF}_{4}$ generations (After Virk, Jinks and Pooni, 1978)*

$\begin{array}{cccccccrr}\text { Variance } & D & H & \text { Variance } & D & H & D_{1} & H_{1} & F_{1} \\ V 1 \mathrm{~F} 3 & \frac{1}{2} & \frac{1}{16} & \text { V1RF3 } & 0 & \frac{1}{8} & \frac{1}{2} & -\frac{1}{16} & -\frac{1}{4} \\ V 2 \mathrm{~F} 3 & \frac{1}{4} & \frac{1}{8} & \text { V2RF3 } & \frac{1}{4} & \frac{1}{8} & 0 & 0 & 0 \\ V 1 \mathrm{~F} 4 & \frac{1}{2} & \frac{1}{64} & \text { V1RF4 } & 0 & \frac{1}{32} & \frac{1}{2} & -\frac{1}{64} & -\frac{1}{8} \\ V 2 \mathrm{~F} 4 & \frac{1}{4} & \frac{1}{32} & \text { V2RF4 } & \frac{1}{4} & \frac{1}{32} & 0 & 0 & 0 \\ V 3 \mathrm{~F} 4 & \frac{1}{8} & \frac{1}{16} & \text { V3RF4 } & \frac{1}{8} & \frac{1}{16} & 0 & 0 & 0\end{array}$

* The definitions of the symbols are those of Virk, Jinks and Pooni (1978).

because they are biased by the effects of unequal gene frequencies and multiple allelism produced by mutation or additional additive and dominance components $\left(D_{1}, H_{1}, F_{1}\right)$ must be fitted to the variances to accommodate these effects.

The detection and analysis of induced mutations using the $F_{2}$ triple test cross design (Kearsey and Jinks, 1968) rests upon the detectable consequences of using inadequate testers (Kearsey and Jinks, 1968; Mather and Jinks, 1971; Virk and Jinks, 1977). For an $F_{2}$ population the parental, $P_{1}$ and $P_{2}$, and $F_{1}$ families from which it arises are ideal testers because the $F_{2}$ population can only segregate for the allelic differences for which $\mathrm{P}_{1}$ and $\mathrm{P}_{2}$ differ, and for which the $\mathrm{F}_{1}$ will be heterozygous. Following irradiation of the $F_{2}$ seed, $P_{1}, P_{2}$ and $F_{1}$ can become inadequate testers in two ways both with different detectable consequences:

(1) Allelic differences can arise at the $k^{\prime}$ loci at which $P_{1}$ and $P_{2}$ do not differ. We shall designate these as common loci.

(2) New alleles, different from those in $\mathrm{P}_{1}$ and $\mathrm{P}_{2}$ can arise at the $k$ loci at which there are already allelic differences between $P_{1}$ and $\mathrm{P}_{2}$. We shall designate these as non-common loci.

For induced genetic differences at the $k^{\prime}$ common loci the triple test cross estimate of $D=\sum_{i=1}^{k} d_{i}^{2}$ becomes

$$
\sum_{i=1}^{k} d_{i}^{2}+\sum_{j=1}^{k^{\prime}} 4 \mu_{j}\left(1-2 \mu_{j}\right)\left(d_{j} \pm h_{j}\right)^{2}
$$

where $\mu_{j}, d_{j}$ and $h_{j}$ are the mutation rate, and the additive genetic and dominance effects generated by the mutation at the $j$ th common locus respectively. The sign of the $h_{j}$ term is positive if the increasing allele mutates to a decreasing allele and is negative if the decreasing allele mutates to an increasing allele. The magnitude of $H=\sum_{i=1}^{k} h_{i}^{2}$ is unchanged by allelic differences arising at the $k^{\prime}$ common loci, and the estimates of epistasis are also unaffected unless these new allelic differences interact with those at the non-common loci.

Induced new alleles at the $k$ non-common loci may deflate or inflate $D, H$ and the epistatic components depending upon whether the additive, dominance and epistatic effects generated by the new alleles are smaller or greater than those generated by the original alleles they replace. The new alleles will always produce a heterozygous combination when crossed to any of the three testers, whilst the original alleles would have produced 
homozygous and heterozygous combinations in equal frequencies. Dominance effects and epistatic interactions involving heterozygous combinations may, therefore, be increased.

In general, therefore, mutations at common loci will affect the estimate of $D$, whilst mutations at non-common loci will also affect the estimates of $H$ and the epistatic components. Unless there are significant differences in the probability of mutations occurring at different loci the relative contributions of mutations at the two types of loci will depend primarily upon the proportion of the loci controlling a character for which $\mathrm{P}_{1}$ and $\mathrm{P}_{2}$ have different alleles i.e., $k: k+k^{\prime}$.

Radiation can also affect the $F_{2}$ population by increasing the recombination rate (Nilan, 1965). It cannot, however, affect any statistic of rank 1 since they are influenced only by recombination events during gametogenesis in the $F_{1}$ which occurred before the treatment was applied (Mather and Jinks, 1971). The estimates of the genetical components from the triple test cross and $V_{1 \mathrm{RF}_{3}}$ and $V_{1 \mathrm{RF}_{4}}$ will therefore be unaffected but the rank 2 and rank 3 statistics derived from $\mathrm{RF}_{3}$ and $\mathrm{RF}_{4}$ generations (table 1) will be affected. Since the predominant linkage phase in the cross of varieties 1 and 5 is known to be repulsion (Jinks and Perkins, 1969; Perkins and Jinks, 1970) these statistics should be inflated by any increased recombination.

\section{EXPERIMENTAL DESIGN}

The experimental material was derived from a random sample of 50 seeds of the $\mathrm{F}_{2}$ of a cross between varieties 1 and 5 of Nicotiana rustica. Twenty five $F_{2}$ seeds were exposed to $20 \mathrm{kr}$ of gamma-radiation from a $\mathrm{Co}^{60}$ radiation source. Preliminary investigations had shown that this dose was high enough to induce mutational changes, whilst producing limited deleterious effects on growth. The remaining 25 unirradiated $F_{2}$ seeds were used to produce the control families.

The $50 \mathrm{~F}_{2}$ plants were raised and examined. No obvious chimaeral segments were found in the irradiated material. All 50 plants were therefore used to set up the following crosses:

(1) Backcrosses onto their parents (V1 and V5) and $F_{1}(V 1 \times V 5)$ produced 24 sets of triple test cross families (Kearsey and Jinks, 1968) from the irradiated $F_{2}\left(R L_{1}, \mathrm{R} L_{2}, \mathrm{R} L_{3}\right)$, and 25 sets from the control $\mathrm{F}_{2}\left(L_{1}, L_{2}, L_{3}\right)$.

(2) The $F_{2}$ plants were selfed to produce $25 \mathrm{~F}_{3}$ families from the control and $25 \mathrm{RF}_{3}$ families from the irradiated plants. Two randomly chosen siblings of each $\mathrm{F}_{3}$ and $\mathrm{RF}_{3}$ family were in turn selfed to produce $50 \mathrm{~F}_{4}$ and $50 \mathrm{RF}_{4}$ families. Twelve individuals of each of the $L_{1}, L_{2}, L_{3}, \mathrm{R} L_{1}, \mathrm{R} L_{2}, \mathrm{R} L_{3}, \mathrm{~F}_{3}, \mathrm{RF}_{3}, \mathrm{~F}_{4}$ and $\mathrm{RF}_{4}$ families were raised in a completely randomised experiment during the summer of 1979. The $F_{3}$ and $R_{3}$ families were a second random sample of siblings from the families which provided the parents of the $F_{4}$ and $\mathrm{RF}_{4}$ families.

Germination was poor for the triple test cross families derived from two of the irradiated $F_{2}$ plants. Only the remaining 22 complete sets of triple test cross families were, therefore, analysed. 


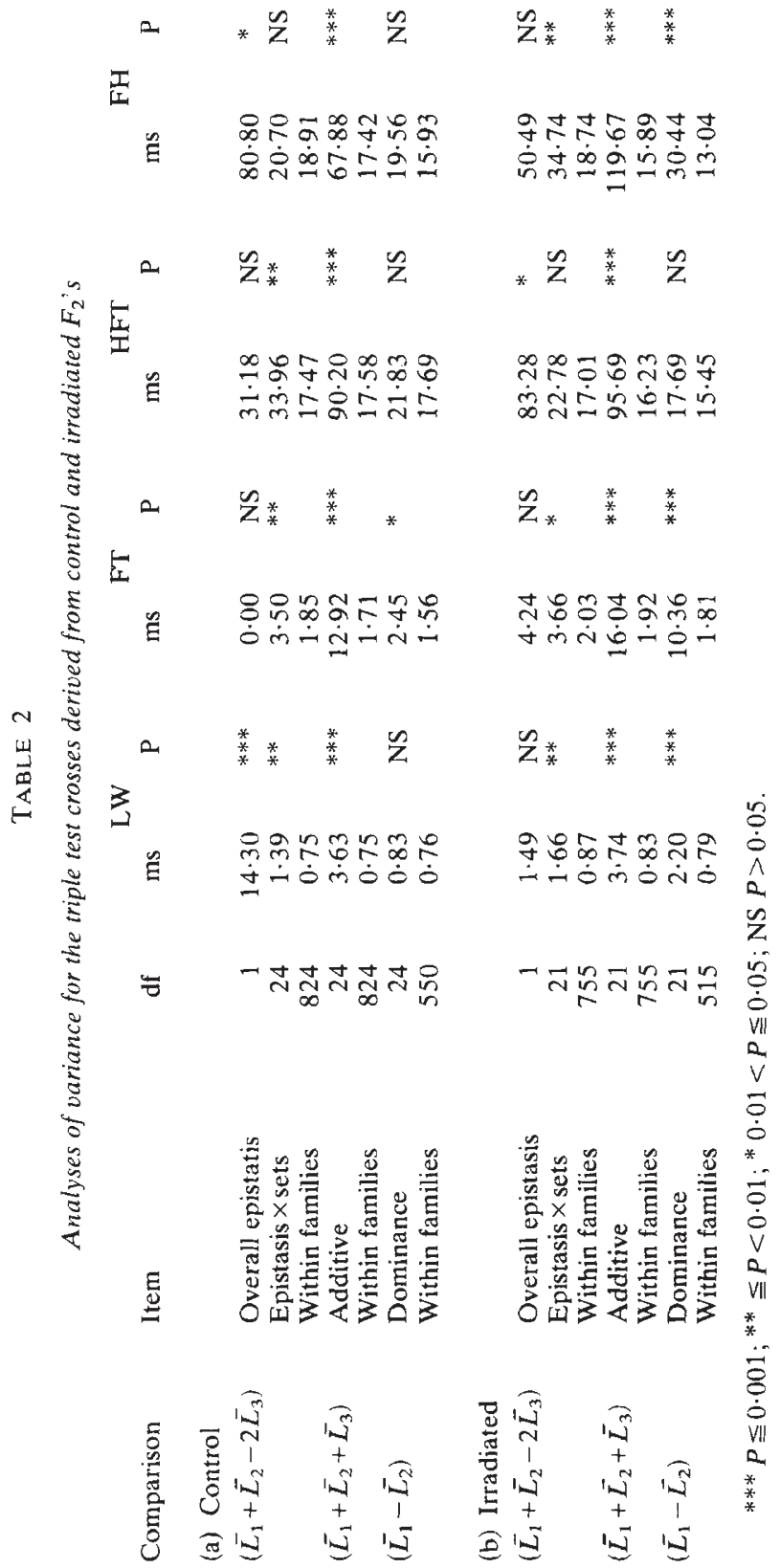


No seed was available for two $\mathrm{F}_{4}$ families and one $\mathrm{RF}_{4}$ family. These families have subsequently been regenerated to avoid gaps in the later generations.

Leaf width (in $\mathrm{cm}$ ) was measured at the widest point of the blade of the largest leaf six weeks after planting. Flowering time was recorded as the number of days to flowering after the $1 \mathrm{July}$. Height at flowering time and the eventual final height of the plants were measured to the nearest $0.5 \mathrm{~cm}$.

\section{REsults}

The two epistasis $\left(L_{1}+L_{2}-2 L_{3}\right)$ items in the triple test cross analyses of the controls (table 2 ) indicate low levels for all four characters. Irradiation appears to have had little effect on the total epistasis present although there are differences in its distribution between the two items (overall epistasis and epistasis $\times$ sets).

The additive $\left(L_{1}+L_{2}+L_{3}\right)$ item is highly significant in all of the analyses (table 2 ) and is consistently larger but not significantly so following irradiation. In the controls the dominance $\left(L_{1}-L_{2}\right)$ item is significant for flowering time only, but it shows a marked increase and is significant for all characters except height at flowering time following irradiation. Estimates of the additive and dominance genetical components derived in the usual way (Kearsey and Jinks, 1968; Perkins and Jinks, 1970) are presented in table 3 .

\section{TABLE 3}

Estimates of the components of variation from triple test cross analyses of the control and irradiated material

\begin{tabular}{|c|c|c|}
\hline Character & Control & Irradiated \\
\hline Leaf width & $\begin{aligned} D & =7.67 \pm 2.80 \\
H & =\mathrm{NS}^{*} \\
E_{w} & =8.06\end{aligned}$ & $\begin{aligned} D & =7.77 \pm 3.08 \\
H & =5.65 \pm 2.72 \\
E_{w} & =8.28\end{aligned}$ \\
\hline Flowering time & $\begin{aligned} D & =29 \cdot 90 \pm 9.95 \\
H & =3 \cdot 56 \pm 2.85 \\
E_{w} & =16 \cdot 31\end{aligned}$ & $\begin{aligned} D & =37.65 \pm 13.20 \\
H & =34.19 \pm 12.80 \\
E_{w} & =14.06\end{aligned}$ \\
\hline Height at flowering & $\begin{aligned} D & =193.66 \pm 69.49 \\
H & =\mathrm{NS}^{*} \\
E_{w} & =182.49\end{aligned}$ & $\begin{aligned} D & =211 \cdot 90 \pm 78 \cdot 78 \\
H & =\mathrm{NS}^{*} \\
E_{w} & =167 \cdot 12\end{aligned}$ \\
\hline Final height & $\begin{aligned} D & =134 \cdot 55 \pm 52 \cdot 30 \\
H & =\mathrm{NS}^{*} \\
E_{w} & =193 \cdot 85\end{aligned}$ & $\begin{aligned} D & =276.74 \pm 98.51 \\
H & =69.59 \pm 37.72 \\
E_{w} & =147.41\end{aligned}$ \\
\hline
\end{tabular}

${ }^{*}$ See Table 2.

Hierarchical analyses of variance for the $F_{3}, F_{4}, R F_{3}$ and $R_{4}$ are presented in table 4 . All items in the analyses are, with one exception ( $V_{1 \mathrm{RF}_{4}}$ for leaf width), significant. The effect of irradiation has been to increase the magnitude of the higher ranking mean squares for all characters except leaf width.

The models based upon the expectations in table 1 were fitted to the five variances available for each character in each treatment using standard weighted least squares procedures. Because of their relatively low 


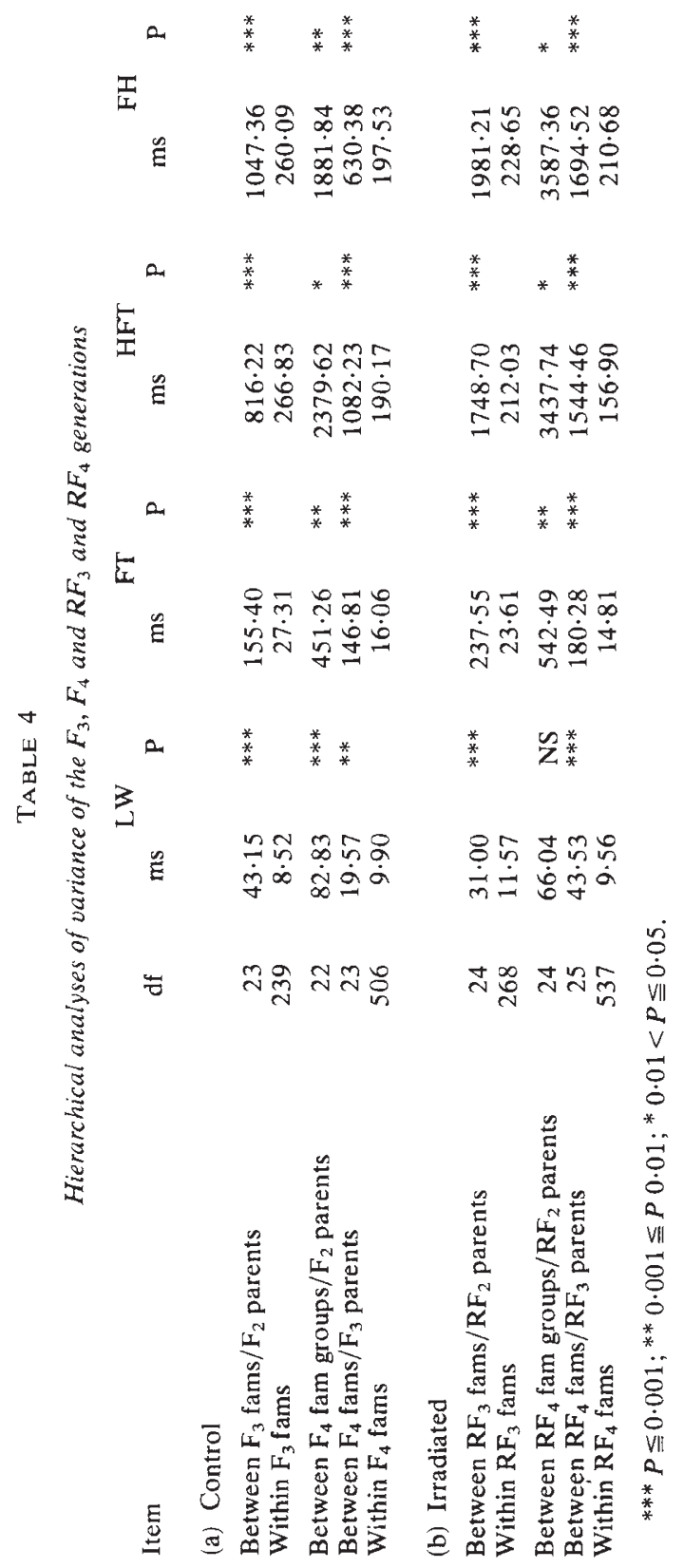


coefficients, there was, as expected, little evidence of a contribution from the dominance components $\left(H\right.$ and $\left.H_{1}\right)$. In fact in all cases a satisfactory model could be found based solely on additive genetic and additive environmental components.

For the controls a simple $D, E_{w}$ model was inadequate for flowering time and height at flowering time (table 5). For these two characters a model which allows for linkage by allowing $D$ to take a different value in the rank 1,2 and 3 statistics $\left(D_{1}, D_{2}\right.$ and $D_{3}$ respectively) was, therefore, fitted and found to be satisfactory. Indeed this model could be reduced to $D_{1}, D_{2}\left(\equiv D_{3}\right)$ and $E_{w}$ without becoming unsatisfactory.

For the irradiated material the simple $D, E_{w}$ model was adequate for all characters except leaf width, which fitted the $D_{1}, D_{2}\left(\equiv D_{3}\right)$ and $E_{w}$ model (table 5). The estimates of the components in these models are not independent, and the value that any component takes depends, to some extent, on the other components being estimated simultaneously. To facilitate comparisons between the control and irradiated material solutions for both the $D, E_{w}$ and $D_{1}, D_{2}, E_{w}$ models are, therefore, given for all characters in table 5 .

In every case the estimate of $D_{2}\left(\equiv D_{3}\right)$ is larger than that of $D_{1}$ but, as expected, only significantly so where the $D, E_{w}$ model is inadequate (FT and HFT for control and LW for irradiated material). For the controls this must be interpreted as repulsion linkage (Mather and Jinks, 1971) whilst for the irradiated material it can arise in the absence of linkage since $D_{1}$, but not $D_{2}(D$ of table 1$)$ is affected by the unequal gene frequencies and multiple allelism generated by the induced mutation. With only two exceptions ( $D_{1}$ for LW and $D_{2}$ for FT) the estimates of $D, D_{1}$ and $D_{2}$ are larger for the irradiated than for the control material, but only significantly so for HFT and FH for $D$, HFT for $D_{1}$ and LW for $D_{2}$. There is, therefore, evidence of a significant increase in the additive genetic variance following irradiation for all characters except flowering time.

\section{Conclusions}

Both the hierarchical inbreeding experiment and the triple test cross detect an increase in the heritable variation following the irradiation of $F_{2}$ seed. In the hierarchical design it appears simply as a marked increase in the additive genetic variance $\left(D, D_{1}\right.$ or $\left.D_{2}\right)$ for all characters except flowering time. The contribution that non-additive genetic components make to the variation in material as inbred as $F_{3}$ and $F_{4}$ is usually too small to be detectable. Not surprisingly, therefore, this design throws no light on these components of the induced variation.

In the triple test cross the increase in the heritable variation is shown by the additive genetic component for flowering time and final height and the dominance component for these characters and for leaf width. There is, however, no marked increase in the epistasis for any character. The interpretation of these increases is not, however, straightforward. The increase in the dominance components reflects solely the result of mutation at the $k$ loci at which the parental varieties ( 1 and 5) already differ. The dominance created by mutation at the other $k^{\prime}$ loci makes its contribution to the additive component only (section 2). It is, therefore, confounded with the genuine additive genetic effects generated by mutation at all $k+k^{\prime}$ 


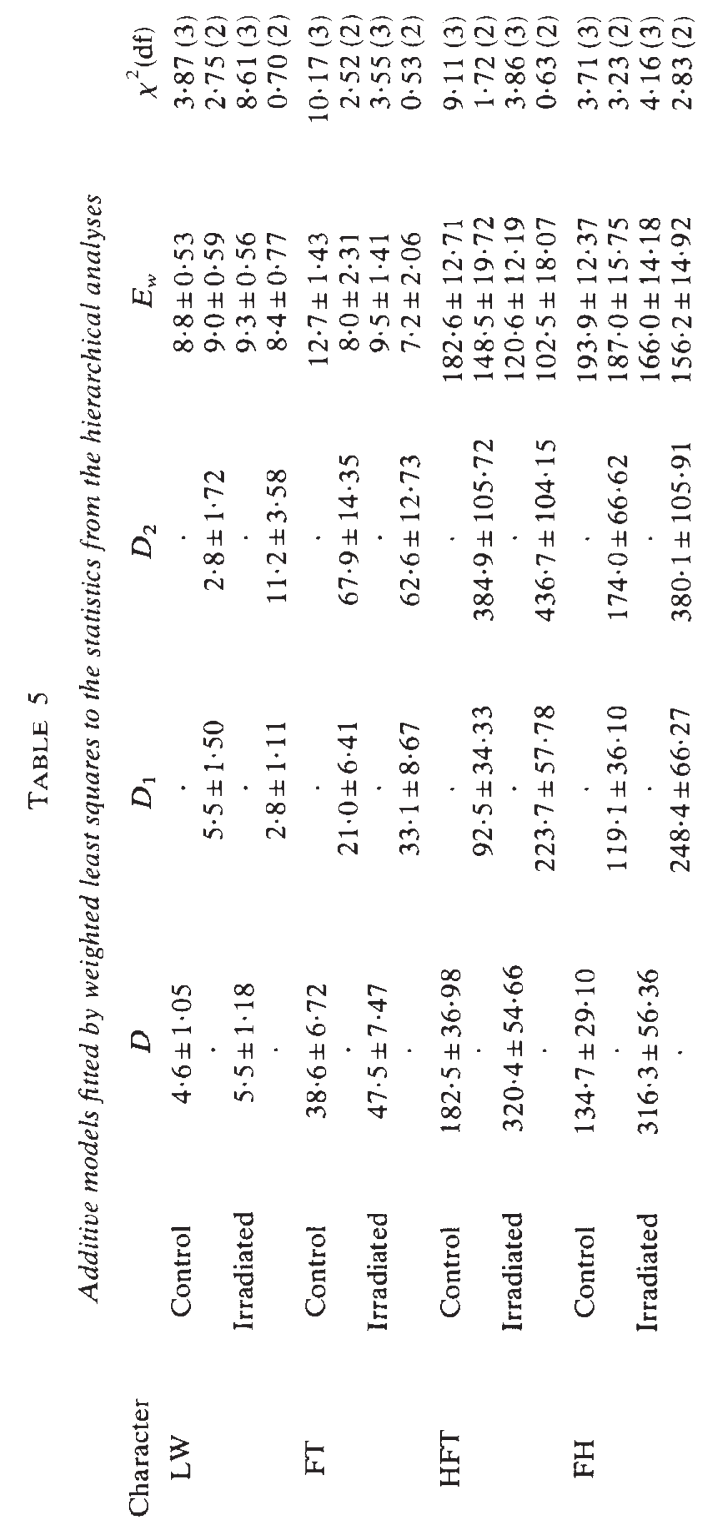


loci. Furthermore, since the dominance contributions at the $k^{\prime}$ loci can take sign depending on the direction of mutation, it can either subtract from or add to the genuine additive contributions to the additive genetic component (section 2).

From the increase in the dominance components we cail therefore conclude that some of the mutations were induced at loci at which the $F_{2}$ was already segregating thus creating three or more alleles at these loci and significantly increasing the dominance but not the epistatic variation. Apart from final height the increases in the additive components are more modest, but as we have already seen they may not be reflecting the true increases in the additive genetic variation produced by mutation.

The estimates of $D$ from the hierarchical and the triple test cross experiments for the control material are in excellent agreement for all characters (tables 3 and 5). For the irradiated material the estimates from the hierarchical experiment are larger for all except leaf width. The smaller estimates from the triple test cross are, of course, what we would expect if they were being reduced by the cancelling effect of dominance created by mutation at the $k^{\prime}$ loci. This is not, however, the only possible explanation. For example, there are differences in the linkage biases of the $D$ 's obtained from the two sources which would make the estimates from the triple test cross the smaller in the presence of repulsion linkages (section 2).

In the control and irradiated material the estimates of $D_{1}$ from the hierarchical experiment are smaller than those of $D_{2}$ as expected in the presence of repulsion linkages (table 5). In addition, the estimates of $D$ from the triple test cross, which are expected to have the same linkage biases as those of $D_{1}$, are generally closer to the latter than to estimates of $D_{2}$ (tables 3 and 5). For the irradiated material, however, $D_{1}$ is also biased by the effects of multiple allelism and unequal gene frequencies created by the induced mutations. We might, therefore, expect the agreement between the estimates of $D$ and $D_{1}$ to be closer for the control than for the irradiated material which is clearly not the case for HFT but appears to be so for LW and FH (tables 3 and 5).

There are therefore some unresolved questions but the main finding, that we can detect an increase in the heritable variation in an $F_{2}$ following irradiation by both of the crossing programmes, is unambiguous. One prediction that follows from this, ramely, that a wider range of pure breeding lines can be extracted from the $\mathrm{F}_{2}$ following irradiation is currently being confirmed.

Acknowledgements.-Financial support is gratefully acknowledged from the Royal Commission for the Exhibition of 1851 (by D.S.V.) and from the S.R.C. (by N.R.I.).

\section{REFERENCES}

KEARSEY, M. J., AND JINKS, J, L. 1968. A general method of detecting additive, dominance and epistatic variation for metrical traits: I. Theory. Heredity, 23, 403-409.

JINKS, J. L., AND PERKINS, J. M. 1969. The detection of linked, epistatic genes for a metrical trait. Heredity, 24, 465-475.

MATHER, K., AND JINKS, J. L. 1971. Biometrical Genetics. 2nd edition. Chapman and Hall, London. 
NILAN, R. A. 1965. Conference Discussion in Lawrence, C. W., and Davies, D. R. The effect of mutagenic agents on recombination in "The Use of Induced Mutations in Plant Breeding." Supplement to Radiation Botany, 5, 769-781.

PERKINS, J. M., AND JINKS, J. L. 1970. Detection and estimation of genotype-environmental, linkage and epistatic components of variation for a metrical trait. Heredity, 25, 157-177. VIRK, D. S., AND JINKS, J. L. 1977. The consequences of using inadequate testers in the simplified triple test cross. Heredity, 38, 237-251.

VIRK, D. S., JINKS, J. L., AND POONI, H. S. 1978. The assessment of induced continuous variation in pure breeding lines following selfing. Heredity, 40, 255-268. 